\title{
Imaginal retraining decreases craving for high- calorie food in overweight and obese women: A randomized controlled trial
}

Steffen Moritz (1) ${ }^{1}$, Anja S. Göritz ${ }^{2}$, Stella Schmotz ${ }^{1}$, Roland Weierstall-Pust ${ }^{3}$, Josefine Gehlenborg ${ }^{1}$, Jürgen Gallinat ${ }^{1}$ and Simone Kühn ${ }^{1}$

\begin{abstract}
Overweight and obesity are epidemic conditions. Obesity is associated with somatic and psychological sequelae, including serious life-shortening disorders (e.g., diabetes). This study aimed to evaluate the efficacy of a newly developed imaginal variant of approach bias modification (i.e., imaginal retraining) for the reduction of craving for high-calorie food. In a randomized controlled trial, 384 women with a body mass index above 25 were allocated to a wait-list control group or to two variants of imaginal retraining (ratio: $1 ; 0.5 ; 0.5$ ). The two intervention groups were sent a manual on imaginal retraining. One group was explicitly encouraged and instructed to use electronic reminders $\left(R_{E R}\right)$; the standard retraining group $\left(R_{S}\right)$ was not encouraged to use electronic reminders. Assessments were 6 weeks apart and were carried out online. Craving for high-calorie food represented the primary outcome (based on the Visual Analog Scale, VAS). Secondary outcomes included the Food Cravings Questionnaire (FCQ-T-R). The study was registered as DRKS00017220. Women in the $R_{E R}$ group utilized the retraining technique more often than those in the $R_{S}$ condition, and utilization frequency in turn was associated with improvement on craving and eating behavior scales. Both intention-to-treat and per-protocol analyses showed a favorable effect of the RER group, which achieved significance on the primary outcome, as well as on several other outcomes relative to controls at a small to medium effect size. For those participants who measured their weight before and after the assessment using a scale, weight loss in the $R_{E R}$ group was significantly greater compared to the control group. Both retraining groups ( $R_{E R}$ : 39.4\%; $R_{S}$ : $31.1 \%)$ reduced their subjective amount of eating relative to controls (24.2\%). Approximately two-thirds of the sample (68.3\%) performed the exercises at least once during the study period. The present results show that, when used regularly, imaginal retraining may reduce craving for high-calorie food in overweight and obese women. Of note, there was also evidence suggestive of weight reduction, although no diet or lifestyle change was recommended in the manual. Because a large subgroup neither read the manual nor performed the exercises, we recommend that future imaginal retraining be conveyed via short video clips.
\end{abstract}

\section{Introduction}

Overweight and obesity are growing problems with epidemic dimensions ${ }^{1,2}$. In Europe, $30.5 \%$ of women and

\footnotetext{
Correspondence: Steffen Moritz (moritz@uke.uni-hamburg.de)

${ }^{1}$ Department of Psychiatry and Psychotherapy, University Medical Center Hamburg-Eppendorf, Hamburg, Germany

${ }^{2}$ Department of Occupational and Consumer Psychology, University of Freiburg, Freiburg, Germany

Full list of author information is available at the end of the article.
}

44.7\% of men are considered obese, and $53.1 \%$ meet the criteria for being overweight ${ }^{3}$. Prevalence is especially high in industrialized countries, but the problem is also on the rise in developing countries ${ }^{1,4}$. Obesity is a primary risk factor for several disorders (e.g., cardiovascular disease, coronary heart disease, sleep apnea, atrial fibrillation, diabetes) and aggravates known risk factors (e.g., high blood pressure) for other life-shortening disorders (e.g., stroke). High BMI is regarded as responsible for 4 million

\section{(c) The Author(s) 2019}

(c) (i) Open Access This article is licensed under a Creative Commons Attribution 4.0 International License, which permits use, sharing, adaptation, distribution and reproduction cc) in any medium or format, as long as you give appropriate credit to the original author(s) and the source, provide a link to the Creative Commons license, and indicate if changes were made. The images or other third party material in this article are included in the article's Creative Commons license, unless indicated otherwise in a credit line to the material. If material is not included in the article's Creative Commons license and your intended use is not permitted by statutory regulation or exceeds the permitted use, you will need to obtain permission directly from the copyright holder. To view a copy of this license, visit http://creativecommons.org/licenses/by/4.0/. 
deaths worldwide per year, representing $7.1 \%$ of deaths due to any cause and contributing to 120 million disability-adjusted life years ${ }^{4}$. Moreover, obesity is associated with psychological sequelae such as (self-)stigma ${ }^{5}$.

Standard measures to decrease weight are dieting (i.e., reducing the intake of calories) and/or increased physical activity to burn additional calories. In severe cases, surgery is recommended ${ }^{6}$ as a last resort despite possible side effects $^{7}$. Some pharmacological agents have also shown promise $^{8}$.

The present article is concerned with another route of intervention-the reduction of craving for food ${ }^{1}$-by means of approach bias modification (ABM). ABM procedures are rooted in dual-process theories ${ }^{9,10}$, which try to explain substance and behavioral addictions, including obesity, as an excess of (implicit) impulsive approach tendencies that override both the (explicit) will to disengage from the substance or behavior and the insight into the negative consequences of the substance or behavior ${ }^{11}$. In line with the tenets of dual-process theories, experimental studies using the approach-avoidance task (AAT) show that individuals with addictive behavior are faster to pull a picture of their preferred/craved substance/object (e.g., drug, alcoholic drink, high-calorie food) toward themselves than to push it away via a joystick in a computerized set-up compared to non-addicted individuals ${ }^{12}$. This effect is well-established across a range of disorders, particularly substance disorders but also obesity (see ref. ${ }^{13}$ for a study with an approach-avoidance variant of the implicit-association task), and speaks to an embodiment of addiction (embodiment or embodied cognition is the theory that many features of an agent's cognition are shaped by aspects of his or her body $\left.{ }^{14}\right)$. Building upon diagnostic findings from the AAT and other assessment procedures, $A B M$ aims to revert and decrease embodiment. The feasibility and effectiveness of the approach is well established in problem drinkers ${ }^{15}$ but has been successfully applied to other addictions as well ${ }^{16}$. In ABM, the addictive substance or behavior is coupled with pushing a joystick, which causes the picture to shrink in size, thus creating the illusion of distance, whereas a positive or neutral cue is coupled with pulling a joystick, which causes the picture to increase in size, thus creating the illusion of approach. Thus, ABM adopts the rationale of the AAT to decrease the approach bias towards addiction-related stimuli. The reduction of relapse in alcohol use disorder is usually small through ABM. Several studies have shown that $A B M$ reduces the exaggerated approach bias in people with addictive behavior, particularly relating to alcohol ${ }^{16-18}$. The approach was

\footnotetext{
[1] Craving for food relates to the strong desire to consume a specific food and is different from normal hunger; craving for food is particularly prevalent for unhealthful food that is high in calories from fat or sugar.
}

then transposed to other addictions, particularly behavioral ones. For eating disorders results are mixed. In one meta-analysis, cognitive bias modification interventions that included ABM resulted in a medium change in the approach bias and small effects on food consumption and cravings for high-calorie food ${ }^{19}$. The latest meta-analysis found no effect of $\mathrm{ABM}$ on eating behavior. Likewise, a recent study, not included in the meta-analysis, found positive effects of $A B M$ on the approach-avoidance bias but not on eating pathology questionnaires or the body mass index $(\mathrm{BMI})^{20}$.

Recent meta-analyses, however, have questioned the general effectiveness of the ABM approach in ameliorating clinical outcomes (addiction and craving) ${ }^{21,22}$. In the meta-analysis by Boffo et al. ${ }^{22}$, cognitive bias modification exerted a small effect on cognitive biases and relapse but not on the reduction of substance use.

The ABM procedure is simple to use, but even its developers acknowledge that the task can be boring and tedious for some individuals ${ }^{23}$. Another obstacle for routine implementation is the need for access to a computer device and a joystick. Moreover, when using computerized devices the available images are limited and difficult to personalize, particularly relating to the typical environment of intake ${ }^{24}$. Further, the many possible combinations of the substance that is craved (e.g., alcohol: wine, beer), brand/type of packaging, and favorite method of consumption (e.g., can, glass, bottle) are hard to capture with pictures.

Imaginal retraining aims to address these limitations. The technique consists of two phases that are easy for the participants to execute. Following a simple negative mood induction to enhance embodiment (for details, see the "Methods" section), the alleged primary mechanism of $\mathrm{ABM}^{25}$, in the averse sequence participants throw (the behavior/movement is actually performed) the imagined high-calorie food away from them. This sequence corresponds to the push movement in conventional ABM. For the opposite sequence, the user engages in a positive mood induction (see the "Methods" section for details) before imagining eating or drinking a delicious but healthy, low-calorie beverage, or food, while coupling this with other positive sensations to enhance the effects of embodiment ${ }^{25}$. This sequence corresponds to the pull movement in conventional ABM. Two randomizedcontrolled trials with problem drinkers ${ }^{24}$ and smokers ${ }^{26}$ showed that imaginal retraining reduced both craving for alcohol/cigarettes (proximal outcome), as well as actual drinking and smoking behavior (distal/clinical outcome).

For the first time, we examined the efficacy of imaginal retraining to reduce craving for high-calorie food (primary outcome) in individuals with overweight $(\mathrm{BMI}>25)$. Secondary outcomes were the Three-Factor Eating Questionnaire (part of the German Fragebogen zum 


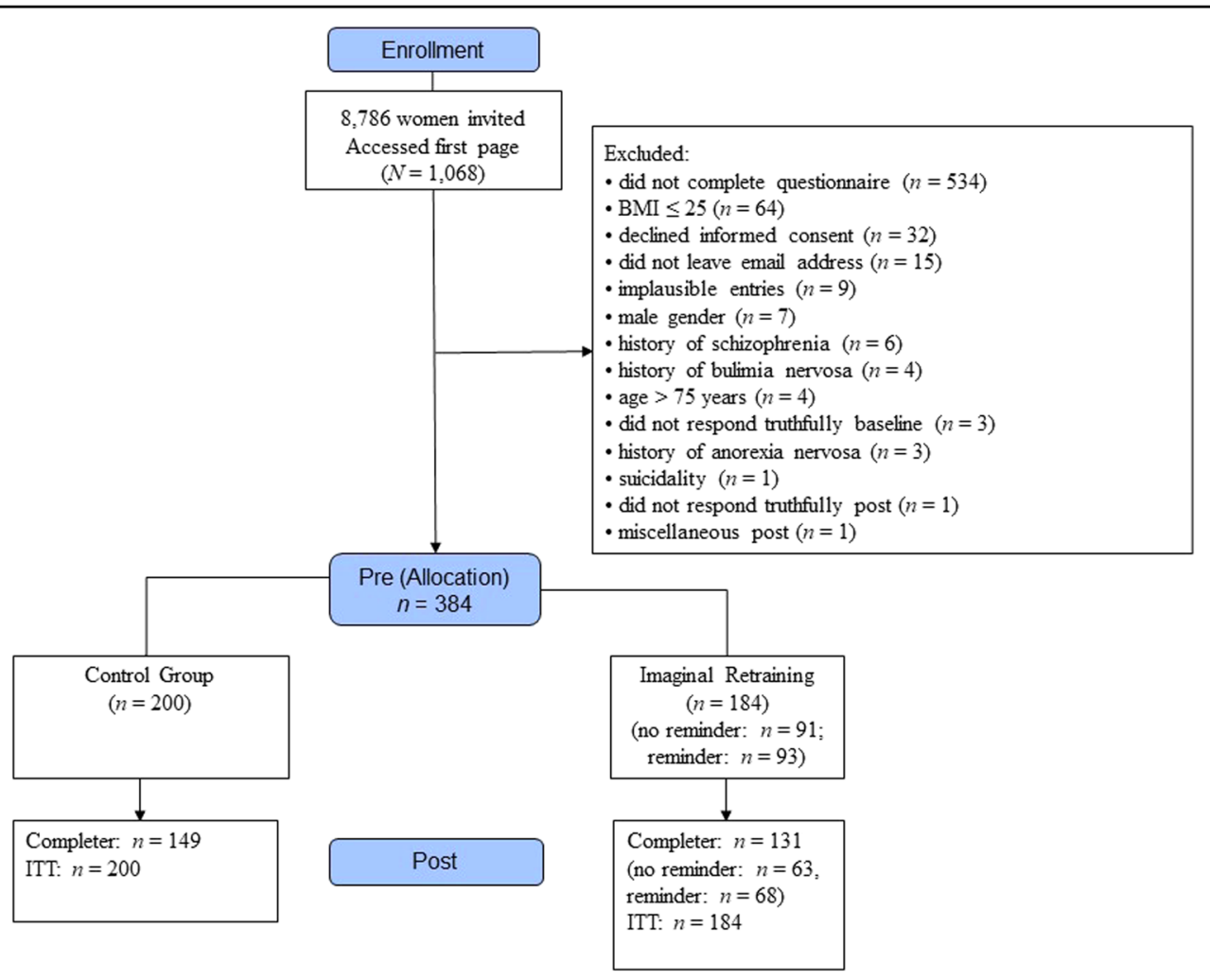

Fig. 1 CONSORT flow chart for the current study. ITT = intention-to-treat analysis.

Essverhalten, FEV; German version ${ }^{27}$ ); the Food Cravings Questionnaire-Trait-reduced (FCQ-T-R $)^{28}$, well-being (WHO Quality of Life scale) ${ }^{29}$, and weight. We adapted the original manual, which we had developed for problem drinkers and smokers, to people with overweight and obesity. The new manual encouraged neither dieting nor any additional physical activity. The (wait-list) control group received access to treatment upon completion of the post assessment.

\section{Methods}

The web-based intervention was conducted via WisoPanel $^{30,31}$, a participant pool of German-speaking individuals who have registered for participation in web-based studies (https://www.wisopanel.net). Members of WisoPanel are drawn from diverse sources comprised of people from all social classes and is demographically similar to the general population.

Because the BMI of women registered with WisoPanel was not known and this was the primary inclusion criterion of the study, all registered women $(N=8.786$ at the time of inclusion) received an invitation and a link to the study. The invited sample thus was comprised of women who were normal weight, overweight, or obese. After a short description of the purpose of the study, interested people were asked to give their explicit informed consent if they met the inclusion criteria (see below). Inclusion criteria were age between 18 and 75 years and a BMI $>25$ (we calculated this based on the weight and height of the participants). A history of anorexia, bulimia, or psychosis led to exclusion. Acute suicidality (as manifested by a score of 2 or 3 on the BDI-II rating for suicidal ideation) led to automatic exclusion with a referral to specific websites offering help, including emergency numbers (see Fig. 1).

\section{Design}

The intervention was unguided; the study was set up as a randomized-controlled trial (randomization plan); controls were allowed access to standard care. Study participation was anonymous; participants were advised to create email addresses that did not disclose their names. Participants in the intervention condition received the imaginal retraining manual immediately after randomization via an email attachment, whereas participants in the control condition received the manual upon completion of the post assessment. One week after the initial sending of the manual, half of the intervention sample received an email with specific instructions on how to set the timer on their smartphone in order to receive daily reminders to perform the exercises (no participant canceled the study nor was 
Table 1 Demographics, well-being, and eating-related characteristics of the sample at baseline.

\begin{tabular}{|c|c|c|c|c|}
\hline & $\begin{array}{l}\text { Wait-list control } \\
(C ; n=200)\end{array}$ & $\begin{array}{l}\text { Retraining standard } \\
\left(R_{S} ; n=91\right)\end{array}$ & $\begin{array}{l}\text { Retraining electronic } \\
\text { reminders }\left(R_{E R}, n=93\right)\end{array}$ & Statistics (df: 2,381 ) \\
\hline \multicolumn{5}{|l|}{ Baseline characteristics } \\
\hline Age in years & $48.79(11.77)$ & $49.35(9.89)$ & $47.77(11.61)$ & $F=0.47, p=0.627$ \\
\hline Diets in parallel in \% & $21.5 \%$ & $20.6 \%$ & $22.1 \%$ & $x^{2}(1)=0.04, p=0.980$ \\
\hline Height in $\mathrm{cm}$ & $166.63(6.71)$ & $165.71(6.62)$ & $166.73(7.21)$ & $F=0.68, p=0.508$ \\
\hline Weight in kg & $90.34(17.31)$ & $89.37(18.56)$ & $88.79(18.70)$ & $F=0.26, p=0.769$ \\
\hline BMI & $32.56(6.13)$ & $32.46(5.99)$ & $31.91(6.15)$ & $F=0.38, p=0.687$ \\
\hline $\begin{array}{l}\text { Obesity (BMI > } 25<30 ; \mathrm{BMI} \geq 30<35 \\
\mathrm{BMI} \geq 35 \text { ) in } \%\end{array}$ & $42.0 / 30.0 / 28.0 \%$ & $39.5 / 34.1 / 26.4 \%$ & $51.6 / 23.7 / 24.7 \%$ & $x^{2}(4)=3.82, p=0.431$ \\
\hline \multicolumn{5}{|l|}{ Eating scales } \\
\hline VAS & $6.74(2.42)$ & $6.45(2.52)$ & $6.60(2.35)$ & $F=0.48, p=0.619$ \\
\hline \multicolumn{5}{|l|}{ FCQ-T-R } \\
\hline Lack of control & $16.56(4.79)$ & $16.47(5.41)$ & $16.71(5.10)$ & $F=0.05, p=0.648$ \\
\hline Thoughts/preoccupation with food & $13.65(5.61)$ & $12.98(5.49)$ & $13.44(6.11)$ & $F=0.43, p=0.649$ \\
\hline Intentions to consume & $6.41(2.16)$ & $6.30(2.27)$ & $6.38(2.22)$ & $F=0.08, p=0.927$ \\
\hline Emotions & $6.75(2.39)$ & $6.63(2.62)$ & $6.95(2.34)$ & $F=0.41, p=0.664$ \\
\hline Triggers & $3.77(1.09)$ & $3.52(1.28)$ & $3.61(1.17)$ & $F=1.59, p=0.206$ \\
\hline Total & $47.13(13.74)$ & $45.89(14.85)$ & $47.09(14.31)$ & $F=0.26, p=0.770$ \\
\hline FEV cognitive restraint & $8.39(4.16)$ & $7.93(4.26)$ & $7.70(4.27)$ & $F=0.95, p=0.388$ \\
\hline FEV disinhibition & $9.72(3.49)$ & $8.90(4.26)$ & $9.78(3.75)$ & $F=1.75, p=0.175$ \\
\hline FEV hunger & $7.66(3.62)$ & 7.05 (3.68) & $7.25(3.45)$ & $F=1.00, p=0.367$ \\
\hline \multicolumn{5}{|l|}{ Psychological scales } \\
\hline $\mathrm{BDI}$ & 14.54 (10.49) & $11.78(8.64)$ & $15.72(9.24)$ & $\begin{array}{l}F=4.02, p=0.019\left(R_{E R}>R_{S}: p=\right. \\
\left.0.007), R_{S}<C: p=0.026\right)\end{array}$ \\
\hline WHOQOL & $3.43(0.87)$ & $3.63(0.83)$ & $3.45(0.79)$ & $F=1.86, p=0.157$ \\
\hline expectancy & $5.48(1.73)$ & $5.19(2.00)$ & $5.51(1.61)$ & $F=1.01, p=0.365$ \\
\hline
\end{tabular}

Means and standard deviations (in brackets)

$B D I-I I$ Beck Depression Inventory II, BMI body mass index, FCQ-T-R Food Cravings Questionnaire-Trait-reduced, FEV Three-Factor Eating Questionnaire (part of the Fragebogen zum Essverhalten, FEV), VAS Visual Analog Scale (craving for high-calorie food), WHOQOL-BREF WHO Quality of Life

excluded from it for not having a smartphone or not being willing to use it).

Data from 384 participants were included in the final analyses (see Table 1), which according to g*power allowed for the detection of a small effect size. The main reasons for exclusion of data were cancellation of the survey before completion, $\mathrm{BMI} \leq 25$, and refusal to give informed consent (see CONSORT flow chart in Fig. 1). The trial was registered with the German Clinical Trials Register (DRKS0001722). We carefully checked that required statistical assumptions were met (e.g., normal distribution; equal variances-for certain comparisons adjustments for unequal variances were made).

\section{Invitation and baseline survey}

Assessments were conducted online using Questback/ UniPark $^{\circledast}$. In keeping with guidelines of the European General Data Protection Regulation (GDPR), no IP addresses were stored. The local ethics committee for psychologists at the University Hospital of Hamburg-Eppendorf (Germany) approved the study (LPEK-0030). As an incentive for participation, a self-help manual on imagery rescripting was sent to all individuals who completed the study ${ }^{32}$, as well as a link to the MCT \& More app (www.uke.de/mct_app), which is aimed to improve self-esteem.
At the start of the survey, electronic informed consent was required. Questions on the participant's sociodemographic background as well as medical history (e.g., prior experience with psychotherapy; current treatment status; prior psychiatric diagnoses, if any) followed. Subsequently, scales on eating behavior and well-being were presented (see section "Questionnaires"). We also asked for the participant's present weight. At the end, we asked participants whether they had truthfully answered the questions (inclusion criterion). We requested a pseudonymized email address. Email addresses were not stored online. Within $24 \mathrm{~h}$, participants were randomized to one of the three conditions (ratio: 1 (wait list); 0.5 standard retraining; 0.5 retraining with electronic reminders). The allocation was implemented based on the time the participant entered the study. Owing to the design of the online study, which involved no personal contact, concealed allocation as in clinical trials with face-to-face assessments could not be implemented. Our procedure is best characterized as centralized assignment with no risk of bias because the person allocating individuals to conditions had no information about participants other than the date they had signed up for the study and their anonymized email.

Participants in the control group were informed via email that they would receive the retraining manual after 
the post assessment. Six weeks after initial participation, all participants were invited to participate in the post assessment. Up to three reminders were sent. For the post assessment, participants were first asked to re-enter their email address to allow matching of pre and post data. The same set of questionnaires was administered as in the baseline survey. We asked participants for their present weight and whether this was measured with a scale (this question was entered during the follow-up period and the results are therefore available for a subgroup; see Tables 2 and 3). Further, we asked whether they had eaten less during the study period vs. the same or more as previously (the latter two categories were later pooled). Those in the retraining conditions who had at least started to read the manual were asked further questions related to their subjective assessment of the quality, comprehensibility, satisfaction, and efficacy of the manual (see Tables 4 and 5).

\section{Imaginal retraining}

Imaginal retraining is a manualized self-help intervention (10 pages with 5 figures; 3918 words). The manual starts with a short psychoeducational section that highlights well-established negative consequences of overweight and obesity in order to increase motivation to change. Next, participants are familiarized with findings using the classical approach-avoidance procedure (diagnostic and intervention $[\mathrm{ABM}]$ procedure). To enhance participants' understanding of the rationale for our procedure, the psychological mechanisms that seem to underlie conventional retraining are explained. Some disadvantages of the original technique (need for access to a computer, lack of personalization, boredom) are identified as issues that our procedure aims to address.

Before conveying the technique of imaginal retraining, we describe exposure in vivo and in sensu, as the latter intervention is an ingrained part of our procedure. Participants are then instructed to imagine their preferred high-calorie foods that they want to eat less of along with their usual environment when eating these foods. Following this, they are asked to imagine a healthy, lowcalorie food they also like to eat (e.g., apples). Participants are informed about the close connection between body posture, thoughts, and emotions. We illustrate that when we are in a bad mood, we tend to walk slumped over and with the corners of our mouths turned down, whereas when we feel happy, we walk with a more upright body posture and have a confident facial expression. Posture and emotion influence each other reciprocally. Thus, straightening the body leads to a slight improvement in mood, whereas adopting a bent-over posture tends to reduce well-being.

Then, imaginal retraining is taught, which consists of two sequences with two steps each. In the first part, the participant is asked to first exhale, slump forward, and round his or her shoulders. This posture should be reinforced as vividly as possible with negative thoughts (i.e., mood induction). Subsequently, participants should imagine their favorite high-calorie food and the place where they usually consume it. Participants should then push or throw the food away from themselves in their imagination (e.g., throwing a cake against the wall) while vigorously executing the movement in reality. This sequence is depicted in the manual by a series of drawings (see Fig. 2). We also advise participants to throw the imagined food onto the ground because pushing away and downward movements are both typically associated with disgust and rejection.

For the approach sequence, participants are instructed to imagine eating healthy food (e.g., an apple). They are asked to take a deep breath and stand up straight and tall as if someone were pulling them up by an imaginary string attached to the top of their head. They are then told to move the imagined healthy food or drink toward their mouth in a somewhat exaggerated, happy manner, as actors often do in advertisements, so that they are looking slightly upwards (to improve mood). At the same time, they should contemplate pleasant thoughts and images (e.g., eating an apple while stroking a pet lying against their chest). Again, drawings are used to depict this procedure (see Fig. 2). Participants are asked to perform the exercises regularly, at least twice a day. No further tips (e.g., regarding a diet or physical exercises) are provided.

After one week, half of the participants in the retraining group (the $\mathrm{R}_{\mathrm{ER}}$ group) were sent specific instructions that included screenshots on programming their android or iOS smartphone to receive messages once or twice daily.

\section{Questionnaires \\ Primary outcome}

Visual Analog Scale (VAS) for food craving. The mean total score of a three-item VAS scale served as the primary outcome. This scale was used in analogous trials in problem drinkers ${ }^{24}$ and smokers ${ }^{26}$. The VAS referred to the previous week. Participants had to move a bar between 0 and 100 to represent the strength of their highcalorie food craving in non-eating phases (not at all $[=0]$ to very strong $[=100]$ ); strongest craving for high-calorie food (not at all $[=0]$ to very strong $[=100]$ ); and frequency of craving for high-calorie food (never $[=0]$ to always $[=100])$. Thus, higher scores indicated stronger craving.

\section{Secondary outcomes}

Food Cravings Questionnaire-Trait-reduced (FCQ-T$R^{28}$ ). The FCQ-T-R is a short version of the Food Cravings Questionnaire-Trait (FCQ- $\mathrm{T}^{33}$. The questionnaire is sensitive to change ${ }^{34,35}$ and is correlated with the BMI, as well as with impulsiveness and is negatively correlated 


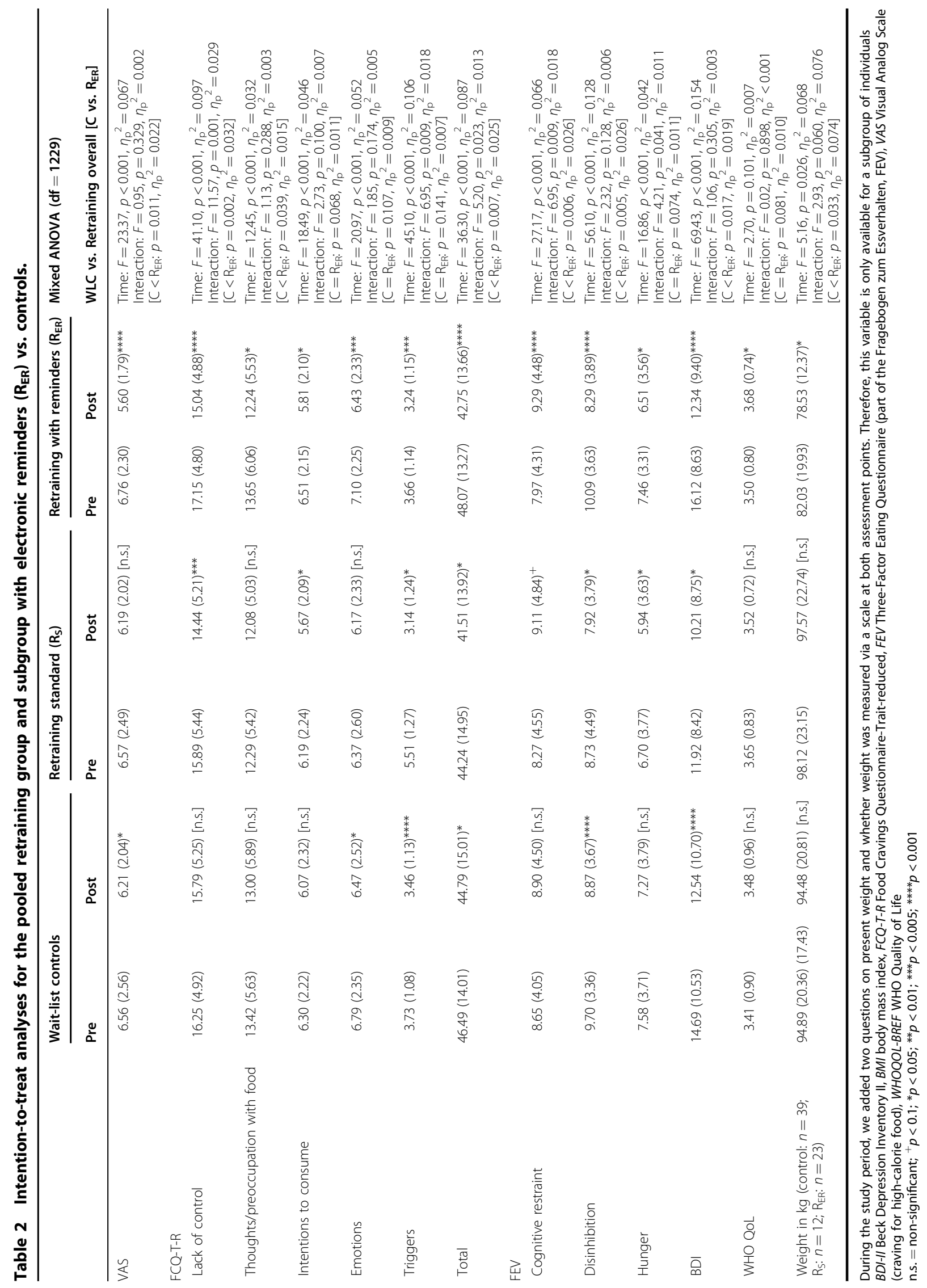




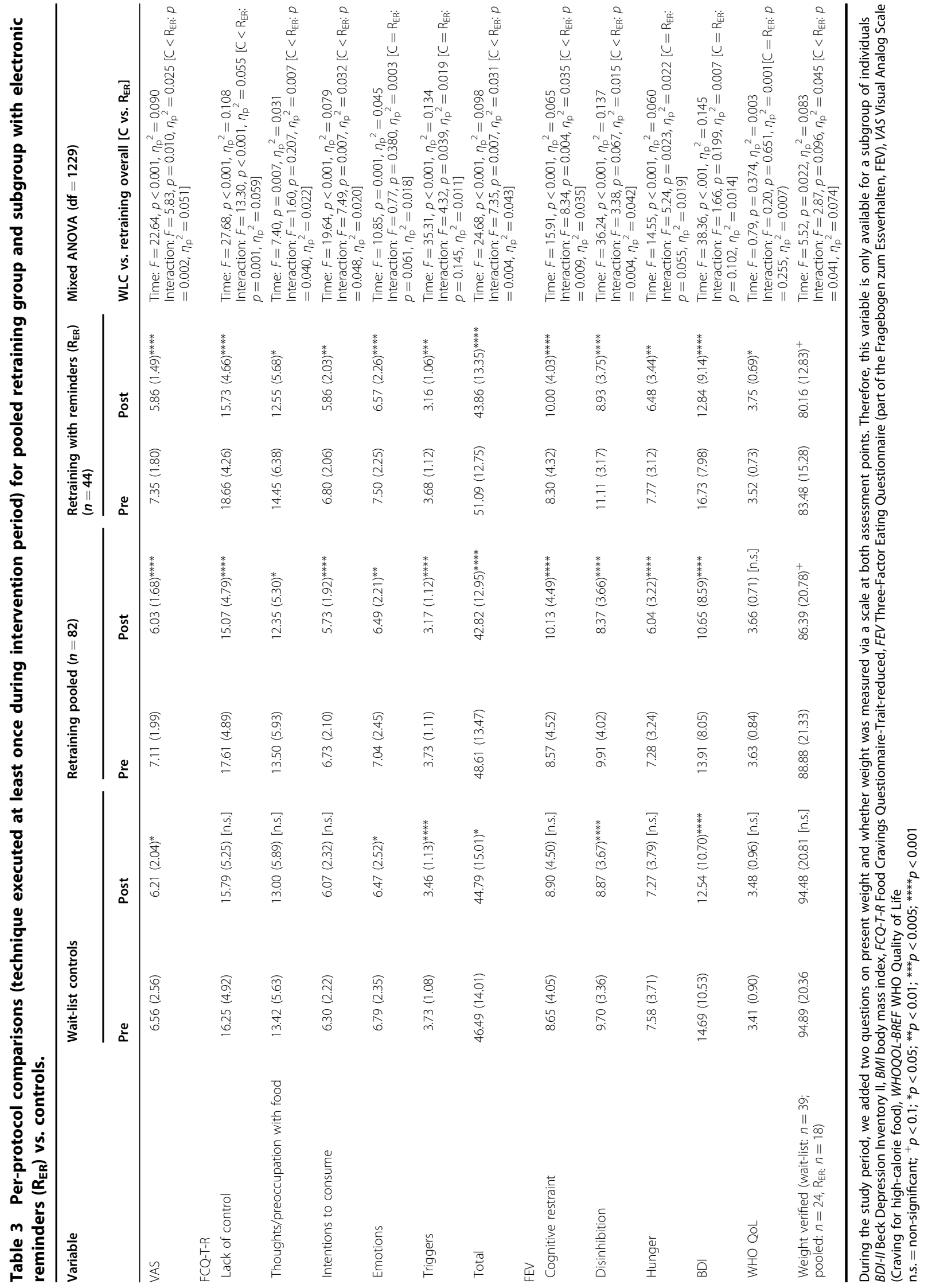


Table 4 Subjective appraisal by users of imaginal retraining (adapted from the Patient Satisfaction Questionnaire, German acronym ZUF-8; Schmidt et al. 1989), with percentage of positive vs. negative appraisals, means and standard deviations (results of the imaginal retraining trial in problem drinkers are inside square brackets).

Item

\begin{tabular}{ll} 
Percentage of positive vs. & negative appraisals; means \\
\hline $\begin{array}{l}\text { Imaginal retraining } \\
\text { standard }\left(R_{S} ; n=38\right)\end{array}$ & $\begin{array}{l}\text { Imaginal retraining with } \\
\text { reminders }\left(R_{E R} ; n=44\right)\end{array}$
\end{tabular}

How do you rate the quality of the manual? (excellent (1), good (2) $81.1 \%$ (2.21 (0.74)) vs. less good (3), not good (4) $)^{\mathrm{a}}$

Did you receive the type of treatment you expected to receive? (not $61.1 \%$ (2.82 (1.09)) at all (1), not really (2) vs. in general, yes (3), yes, absolutely (4))

To what extent did the manual help you cope with your problems? $51.4 \%(2.76(1.00))$ (it helped me cope with almost all of my problems (1), it helped me cope with the majority of my problems (2) vs. it helped me very little (3), it did not help me at all (4)) ${ }^{\mathrm{a}}$

Would you recommend the manual to a friend with similar symptoms? (definitely not (1), probably not (2) vs. probably yes (3), absolutely (4))

How happy are you about the extent of the help you have received through using the manual? (dissatisfied (1), somewhat dissatisfied (2) vs. mostly satisfied (3), very satisfied (4))

Did the manual help you cope with your problems more successfully? (yes, it absolutely helped me (1), yes, it helped me a little (2) vs. no, it did not help me that much (3), no, it did not help me at all (4)) $)^{\text {a }}$

How satisfied are you with the manual in general? (very satisfied (1), $70.3 \%(2.34(0.99)$ mostly satisfied (2) vs. somewhat unsatisfied (3), unsatisfied (4)) ${ }^{a}$

Would you use the manual again? (definitely not (1), probably not $63.2 \%$ (2.87 (1.02)) (2) vs. probably yes (3), yes (4))

Statistics

$\begin{array}{ll}92 . \%(2.18(1.06)) & x^{2}(1)=2.22, p=0.136 \\ & {[81.8 \%][2.05(0.58)]} \\ & x^{2}(1)=4.60, p=0.032 \\ & {[72.7 \%][2.86(0.77)]} \\ & x^{2}(1)=1.47, p=0.225 \\ 65 \%(2.61(0.97)) & {[59.1 \%][2.55(0.91)]}\end{array}$

$\begin{array}{lll}73.7 \%(3.00(0.93)) & & x^{2}(1)=1.80, p=0.179 \\ & {[77.3 \%][3.14(0.99)]} \\ 77.1 \%(3.05(1.04)) & & \\ & & x^{2}(1)=0.00, p=0.971 \\ & {[68.2 \%][2.91(0.87)]} \\ 58.3 \%(2.45(0.95)) & & \\ & & x^{2}(1)=1.50, p=0.221 \\ & {[86.4 \%][1.91(0.87)]} \\ 70.3 \%(2.34(0.99) & & \\ & & \\ 63.2 \%(2.87(1.02)) & & x^{2}(1)=0.860, p=0.354 \\ & & {[77.3 \%][2.09(0.87)]} \\ & & x^{2}(1)=4.61, p=0.032 \\ & & {[77.3 \%][3.09(1.15)]}\end{array}$

aLower scores indicate more positive values (inverted scores)

Table 5 Subjective appraisal of the retraining manual, with means and standard deviations (results from problem drinkers are inside square brackets).

\begin{tabular}{|c|c|c|c|}
\hline Item & $\begin{array}{l}\text { Imaginal retraining standard } \\
\left(\mathrm{R}_{\mathrm{S}} ; n=38\right)\end{array}$ & $\begin{array}{l}\text { Imaginal retraining with } \\
\text { reminders }\left(R_{E R} ; n=44\right)\end{array}$ & $\begin{array}{l}\text { Endorsement in \% (fully } \\
\text { applies through applies a little) }\end{array}$ \\
\hline $\begin{array}{l}\text { I think the retraining manual is good for self- } \\
\text { help and self-guidance. }\end{array}$ & $92.1 \%(3.08(0.97))$ & $97.7 \%(3.02(0.88))$ & $\begin{array}{l}x^{2}(1)=1.39, p=0.239[100 \%] \\
{[2.91(0.75)]}\end{array}$ \\
\hline $\begin{array}{l}\text { My consumption of high-calorie food has } \\
\text { decreased because of the application of the } \\
\text { program. }\end{array}$ & $63.2 \%(2.13(1.07)$ & $79.5 \%(2.09(0.77)$ & $\begin{array}{l}X^{2}(1)=2.713, p=0.100[72.7 \%] \\
{[2.41(1.10)]}\end{array}$ \\
\hline $\begin{array}{l}\text { I think the content of the manual was } \\
\text { comprehensible. }\end{array}$ & $100 \%(3.58(0.64))$ & 100\% (3.52 (0.70) & n/a [100\%] [3.55 (0.60)] \\
\hline I think the manual was helpful. & $86.8 \%(2.68(1.02))$ & $93.2 \%(2.91(0.88)$ & $\begin{array}{l}X^{2}(1)=0.931, p=0.335[90.9 \%] \\
{[2.91(0.97)]}\end{array}$ \\
\hline $\begin{array}{l}\text { I was able to use the manual on a regular } \\
\text { basis during the past six weeks. }\end{array}$ & $55.3 \%(1.95(0.98))$ & $70.5 \%(2.25(1.04))$ & $\begin{array}{l}X^{2}(1)=2.028, p=0.154[86.4 \%] \\
{[2.73(1.03)]}\end{array}$ \\
\hline I had to force myself to use the manual. & $76.3 \%(2.74(1.18))$ & $84.1 \%(2.45(0.98))$ & $\begin{array}{l}x^{2}(1)=0.785, p=0.376[68.2 \%] \\
{[2.00(0.82)]}\end{array}$ \\
\hline $\begin{array}{l}\text { I think the manual would make more sense if } \\
\text { it were used in combination with } \\
\text { psychotherapy. }\end{array}$ & $81.6 \%(2.84(1.08))$ & $93.2 \%$ (3.02 (0.93) & $\begin{array}{l}x^{2}(1)=2.564, p=0.109[95.5 \%] \\
{[3.00(0.82)]}\end{array}$ \\
\hline $\begin{array}{l}\text { The manual is not applicable to my eating } \\
\text { behavior. }\end{array}$ & $55.3 \%(1.95(1.06)$ & $52.3 \%(1.84(0.99)$ & $\begin{array}{l}x^{2}(1)=0.73, p=0.787[40.9 \%] \\
{[1.68(0.95)]}\end{array}$ \\
\hline
\end{tabular}

Note: Scoring: $1=$ not at all, $2=$ a little, $3=$ a lot, $4=$ absolutely, $\mathrm{n} / \mathrm{a}=$ not applicable 


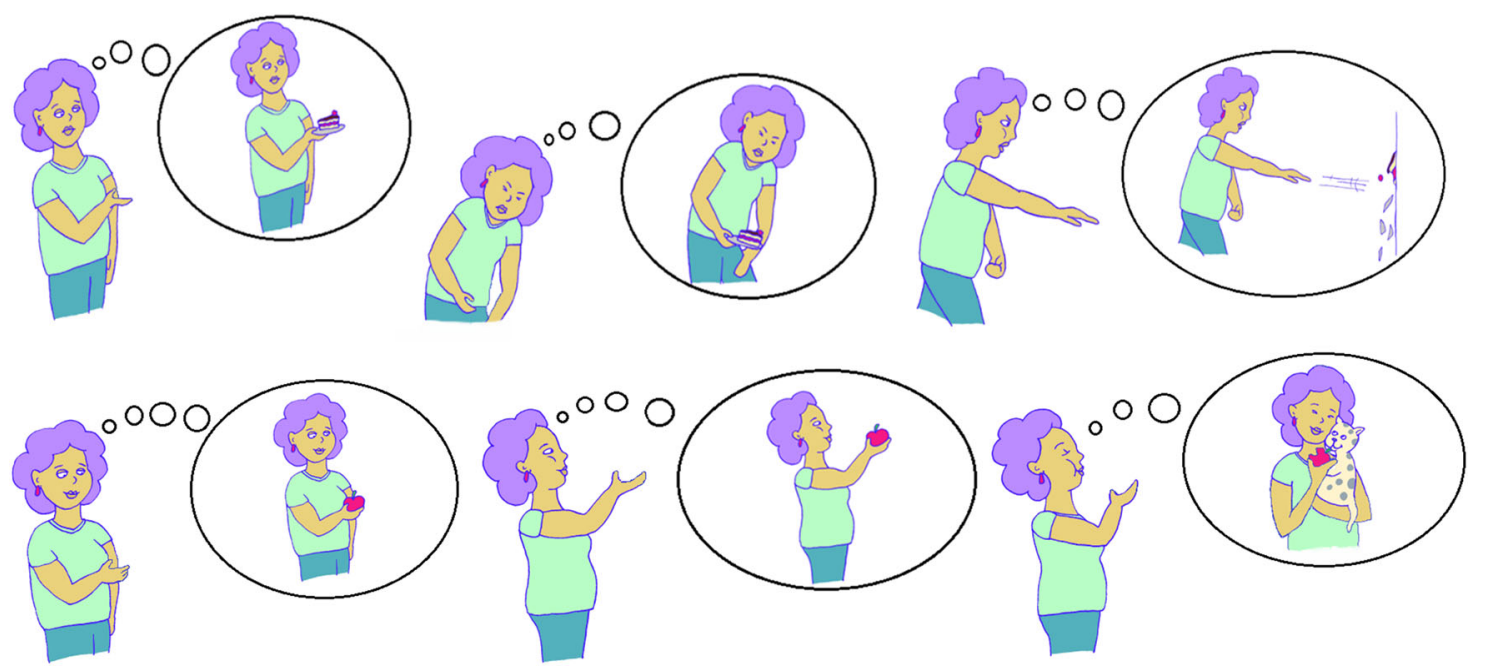

Fig. 2 The two steps of imaginal retraining. Aversion sequence (upper panel): The individual should imagine grabbing a favorite high-calorie food, bend forward and contemplate negative thoughts, and then throwing the imagined food away from themselves (actual movement with imagined object). Approach sequence (lower panel): The individual should imagine grabbing a healthy food such as an apple, stand upright, lift the imagined food high and, if possible, couple this with other positive feelings (e.g., stroking a pet; actual movement with imagined object).

with success in $\operatorname{diets}^{28}$. The short form consists of five subscales: lack of control over eating (five items; Lack of Control), thoughts or preoccupation with food (five items; Thoughts/Preoccupation with Food), intentions and plans to consume food (two items; Intentions to Consume), emotions before or during food craving (two items; Emotions), and cues that may trigger food craving (one item; Triggers). Following Holmes and Meule ${ }^{36}$, items have to be endorsed on a 5-point Likert scale. Internal consistency $(\alpha=0.94)$ as well as test-retest reliability $(r=$ $0.74)$ are $\operatorname{good}^{28,37}$. Higher scores indicate greater problems with eating behavior.

Three-Factor Eating Questionnaire (Fragebogen zum Essverhalten, $\mathrm{FEV}^{27}$ ). The German FEV is a multidimensional questionnaire and incorporates the English Three-Factor Eating Questionnaire (TEFQ) by Stunkard and Messick $^{38}$, a commonly used instrument ${ }^{39}$. Three subscales exist. The first is cognitive restraint from eating (21 items). A high score represents spontaneous and virtually uncontrolled binge eating; a low score hints at controlled eating by means of, for example, counting calories (e.g., "I have a pretty good idea of the number of calories in common foods"; "I consciously hold back at meals in order not to gain weight"). The subscale disinhibition is composed of 16 items. A high score shows cuedriven excessive eating behavior due to, for example, emotional turmoil and external triggers (e.g., "I usually eat too much at social occasions, like parties and picnics"; "When I feel anxious, I find myself eating"). The third scale taps hunger. A high score indicates bothersome sensations of hunger (e.g., "I am usually so hungry that I eat more than three times a day"; "Dieting is so hard for me because I just get too hungry"). For the German version $^{27}$, high validity and internal consistency has been reported (Cronbach's $\alpha=0.74-0.87$ ).

Beck Depression Inventory II $\left(B D I-I^{40}\right)$. The BDI-II contains 21 items that capture common somatic and psychological symptoms of depression. The internal consistency and test-retest reliability of the German version are good ${ }^{41}$.

WHO Quality of Life (WHOQOL-BREF ${ }^{29}$ ). The global item of the WHOQOL-BREF served as an index of quality of life.

\section{Subjective appraisal and benefit}

Participants who had at least started to read the manual were asked to fill out the Patient Satisfaction Questionnaire (German acronym ZUF- $8^{42}$, adapted for online interventions). The scale assesses subjective appraisal of the technique (e.g., quality, satisfaction, effectiveness, and intention to use the application in the future). Tables 4 and 5 show the results of these and additional questions pertaining to treatment.

\section{Results}

The final sample consisted of 384 women with a BMI > 25. A total of 200 participants were allocated to the control condition, while 91 were allocated to standard retraining $\left(R_{S}\right)$ and 93 to retraining with electronic reminders $\left(\mathrm{R}_{\mathrm{ER}}\right)$. Baseline sociodemographic and eatingrelevant characteristics of the three conditions are displayed in Table 1. The participants were in their late 40s. The mean BMI corresponds to obesity, and the mean BDI-II suggests the presence of minimal to mild depressive symptoms. Approximately one-third of the sample 
(33.1\%) had attempted one to three diets in the past. Almost one-fifth (20.5\%) had attempted more than nine diets or were on a permanent diet. Approximately the same percentage had never tried a diet (20.6\%).

Adherence was comparable across groups (controls: 74.5\%; standard imaginal retraining without electronic reminders $\left[\mathrm{R}_{\mathrm{S}}\right]: 69 \%$; retraining with electronic reminders $\left.\left[\mathrm{R}_{\mathrm{ER}}\right]: 73 \%\right), \chi^{2}(2)=0.88, p=0.643$. Being on a diet during the study period did not influence either craving or weight and did not interact with intervention in the two-way ANOVA with Group and Diet (yes, no) as factors $(p>0.2)$

Of those in the two retraining conditions who agreed to rate their degree of engagement with the treatment, 5\% acknowledged that they had not read the manual at all (prior study on smoking: $8.5 \%$ ), whereas $26.7 \%$ reported they had read the manual at least partially but had not (yet) performed any exercises (prior study: 43.9\%); 18.3\% had performed the exercises once in the intervention period (prior study: $24.4 \%$ ); and $17.5 \%$ had performed the exercises once a week (prior study: 24.4\%). Almost onefourth $(24.2 \%)$ had performed the exercises several times a week (prior study $17.1 \%$ ). Six participants (5\%) had performed the exercises on a daily basis (prior study: 1.2\%). Few participants (3.3\%) had performed the exercises several times daily (prior study: 4.9\%). Thus, approximately two-thirds of the sample $(68.3 \%)$ had performed the exercises at least once during the study period.

Of the controls, $24.2 \%$ (prior study on smoking: $26.4 \%$ ) self-reported eating less during the intervention period compared to $36.1 \%$ in the $\mathrm{R}_{\mathrm{S}}$ and $39.4 \%$ in the $\mathrm{R}_{\mathrm{ER}}$ conditions (prior study on smoking: $45.5 \%), \chi^{2}(2)=6.19, p=$ 0.045 . The latter rates rose to $45.9 \%$ and $46.5 \%$, respectively, when considering only those who had performed the exercises at least once in the treatment period (prior study: $59 \%), \chi^{2}(1)=11.70, p=0.003$.

\section{Utilization and dose effects}

Participants in the standard $R_{S}$ condition used the technique somewhat less often than those in the $R_{\mathrm{ER}}$ condition who were encouraged to use electronic reminders (criterion: usage multiple two times a week or more often: $24.1 \%$ vs. $40.3 \%), \chi^{2}(1)=3.58, p=0.059$.

The frequency of performance of the technique (i.e., only read manual; performed exercises once during the study period; performed once weekly; performed several times a week; performed daily; performed several times daily) correlated with changes on certain eating-related parameters suggestive of a beneficial effect of the intervention on outcome: VAS $(r=0.22, p=0.015)$, FCQ-T-R Total $(r$ $=0.35, p<0.001)$, FCQ-T-R Lack of Control $(r=0.37, p<$ $0.001)$, FCQ-T-R Thoughts/Preoccupation with Food $(r=$ $0.28, p=0.002)$, FCQ-T-R Intentions to Consume $(r=$ $0.33, p<0.009)$, FEV Cognitive Restraint $(r=-0.24, p<$ $0.009)$, and FEV disinhibition $(r=0.23, p<0.012)$.

\section{Test-retest reliability}

Test-retest reliability was satisfactory to good, with the following scores (in ascending order of strength): weight $(r=0.98)$, BDI-II $(r=0.79)$, FEV Cognitive Restraint $(r=$ $0.72)$, and FCQ-T-R Total ( $r=0.70$; FCQ-T-R subscales: $r=0.56-0.73)$. Reliability of FEV Disinhibition $(r=0.69)$, FEV Hunger $(r=0.66)$, the QoL global item $(r=0.60)$, and the VAS composite score $(r=0.49)$ were acceptable (all $p<0.001$ ).

\section{Intention-to-treat and per-protocol analyses}

Group differences across time were calculated using mixed ANOVAs with Group as the between-subjects and Time as the within-subject factor. The intention-to-treat (ITT; missing values were estimated using the expectation-maximization method) and the withinsubject analyses results are given in Table 2. Except for quality of life, participants improved on all scores, including depression, irrespective of group allocation. The pooled imaginal retraining group displayed greater improvements than controls on four outcomes: FEV Cognitive Restraint, FEV Hunger, FCQ-T-R Total, and FCQ-T-R Lack of Control. Yet, no differences emerged on the VAS, the primary outcome. When analyses were confined to the retraining group with electronic reminders $\left(R_{E R}\right)$, which showed better adherence than the standard $\left(R_{S}\right)$ group, seven outcomes, including the primary outcome, yielded significance: VAS, FEV Cognitive Restraint, FEV Disinhibition, FCQ-T-R Total, FCQ-T-R Lack of Control, FCQ-T-R Thoughts/Preoccupation with Food, and BDI-II (results pertaining to the BDI-II should be interpreted cautiously in view of baseline differences). Effect sizes for significant results were in the small to medium range. In the subgroup in which weight was measured with a scale, the pooled retraining group showed a greater weight loss at statistical trend level, which achieved significance when considering the $R_{E R}$ group only (see Table 2).

Results were corroborated by the per-protocol (PP) analyses, considering only those participants who had used the technique at least once in the treatment period. Again, participants improved overall at a medium to large effect size except for quality of life. The pooled retraining group was superior to the control group on seven out of 12 outcomes: VAS, FEV Cognitive Restraint, FEV Hunger, FCQ-T-R Total, FCQ-T-R Lack of Control, FCQ-T-R Intentions to Consume, and FCQ-T-R Triggers. At statistical trend level, weight loss was more marked in the pooled retraining group when considering those who endorsed that they had measured their weight using a scale at both baseline and reassessment. When constricting analyses to the $R_{E R}$ group, this outcome achieved significance at a medium effect size. Again, for seven outcomes the $\mathrm{R}_{\mathrm{ER}}$ group had better results than controls; 
two results bordered on significance and three were nonsignificant.

The two retraining subgroups performed almost equally except on the QoL $\left(p=0.016, \eta_{\mathrm{p}}{ }^{2}=0.070\right)$ and FCQ-T-R Emotions $\left(p=0.042, \eta_{\mathrm{p}}{ }^{2}=0.051\right)$, where the $\mathrm{R}_{\mathrm{ER}}$ displayed grater improvement. The paired comparisons indicate that group differences were due to improvement in the experimental conditions and not worsening in the control condition. In fact, controls improved on most outcomes across time.

\section{Completers vs. non-completers}

Compared to non-completers, completers were older ( $p$ $=0.017$ ), more often endorsed that they had attempted more than 15 diets $(p<0.001)$, had somewhat higher treatment expectancy $(p=0.044)$, and scored higher on FEV Cognitive Restraint $(p=0.039)$.

\section{Subjective appraisal}

Tables 4 and 5 display the subjective appraisals of the participants in the two retraining groups who had performed the exercises at least once. Appraisals were better for the group with electronic reminders on the following parameters: whether they had received the expected treatment and whether participants would use the manual again. At trend level, the electronic reminder group more often endorsed that their consumption of high-calorie food had decreased because of their use of the program $(79.5 \%$ vs. $63.2 \%)$. More than $80 \%$ of the participants endorsed the following statements: good or excellent quality of the manual, would recommend the manual to a friend ( $R_{E R}$ only), good for self-help, comprehensible, the manual would have made more sense if it were used in combination with psychotherapy, I had to force myself to use the manual ( $R_{E R}$ only), expected type of treatment received ( $R_{E R}$ only), and helpful and would use manual again ( $R_{E R}$ only). Between $70 \%$ and $79.9 \%$ endorsed the following items: I had to force myself to use the manual ( $R_{S}$ only), helped me cope with problems more successfully $\left(R_{E R}\right.$ only), satisfied with the manual in general, happy about the extent of help received from the manual, consumption of high-calorie food decreased because of the program ( $R_{E R}$ only), able to use the manual on a regular basis ( $\mathrm{R}_{\mathrm{ER}}$ only), and would recommend the manual to a friend ( $R_{S}$ only). Between $60 \%$ and $69.9 \%$ endorsed the following statements: would use manual again $\left(R_{S}\right)$, consumption of high-calorie food decreased because of the program ( $R_{S}$ only), helped me cope with problems ( $R_{E R}$ only), and expected type of treatment received ( $R_{S}$ only).

For use as a benchmark, the figures from our forerunner trial on imaginal retraining in problem drinkers are set in square brackets in Tables 4 and 5 . We comment on the differences to the benchmark if they exceed $10 \%$. More participants in the current trial $\left(\mathrm{R}_{\mathrm{ER}}: 52.3 \%, \mathrm{R}_{\mathrm{S}}: 55.3 \%\right)$ endorsed that the manual was not applicable to their problem behavior than in the alcohol study (40.9\%). In particular, the participants in the $\mathrm{R}_{\mathrm{S}}$ condition endorsed less often that they were able to use the manual on a regular basis during the previous 6 weeks $(55.3 \%$ vs. $86.4 \%$ in the prior study). Again, less participants in the current trial ( $\left.\mathrm{R}_{\mathrm{ER}}: 71.8 \%, \mathrm{R}_{\mathrm{S}}: 58.3 \%\right)$ endorsed that the manual helped them cope with their problems more successfully relative to the alcohol study (86.4\%). Yet, their happiness about the help they received was markedly higher.

\section{Discussion}

Individuals intending to lose weight usually either start a diet aimed at the reduction of calorie intake or initiate/ enhance recreational activities (e.g., sports) aimed at burning additional calories. Such measures necessitate effort and discipline and are often short-lived. Once they end, the weight often increases ${ }^{43,44}$, sometimes above baseline due to hormonal changes (i.e., the yo-yo effect $^{45}$ ).

We borrowed the present technique from the computerized ABM paradigm, which has frequently been administered in studies related to eating ${ }^{20,46}$, but we pursued another route. We expected the intake of and craving for high-calorie food to be reduced by a simple, quick technique that dampens the craving/addiction-like properties of conditioned high-calorie food stimuli. Participants learn to couple the image of their favorite unhealthy/high-calorie food with a mildly aversive emotion (mood induction) and a push movement, similar to classical $A B M$ but with no need for technical equipment $^{24}$. This imaginal variant of retraining has been successfully tested in problem drinkers ${ }^{24}$ and smokers ${ }^{26}$, and the technique reduced craving and also actual consumption. The present study transposed the approach to food.

Unique to this study, two retraining conditions were set up to test whether adherence could be improved by reminders. Similar to the forerunner studies, one group, the standard retraining $\left(R_{S}\right)$ group, received the manual with instructions to read through the manual and to perform the exercises on a daily basis. The other group $\left(R_{E R}\right)$ received the same manual but was also sent an email after one week with specific instructions on how to use the timer on their smartphone in order to give them daily reminders to perform the exercises. This condition was implemented following evidence that daily smartphone notifications to perform simple exercises foster reduction of target symptoms ${ }^{47}$. Indeed, the latter group performed the exercises more regularly, and this frequency in turn was associated with improvement on several outcomes at a small to medium effect size. Accordingly, overall effects were larger for the $R_{E R}$ group than for the other retraining 
group. Both ITT and PP analyses indicate that this subgroup benefited from the intervention in terms of craving and also psychopathological symptoms (depression). For the small subgroup who had measured their weight before and after the study period with a scale, weight loss was more than $3 \mathrm{~kg}$, which represented a medium effect size relative to controls. The pooled retraining group showed a significant effect on craving in the PP but not the ITT analyses. Effects on five outcomes, however, were significant for both kinds of analyses: FCQ-T-R Lack of Control, FCQ-T-R Triggers, FCQ-T-R Total, FEV Cognitive Restraint, and FEV Hunger.

Individuals in both retraining groups reported eating less than controls during the intervention period $\left(\mathrm{R}_{\mathrm{ER}}\right.$ : $39.4 \%$; $\mathrm{R}_{\mathrm{S}}: 36.1 \%$ vs. Control: $24.2 \%$ ), and the majority would use the training again and recommend it to others. Adherence was larger than in smokers. Satisfaction was less for a number of the outcomes relative to the study on alcohol (e.g., able to use the manual on a regular basis, helped me cope with problems). Overall, $R_{E R}$ received more favorable assessments than $R_{S}$, which reached significance for two outcomes. The appraisal of the $\mathrm{R}_{\mathrm{ER}}$ technique in individuals with overweight was numerically better on 14 of the items relative to $R_{S}$ and on 11 of the outcomes compared to the treatment manual for problem drinkers.

Effects for participants in the current study were significant on core outcomes but somewhat weaker than for smokers and drinkers. At this point, we can only speculate about possible reasons. First, smaller effects were likely due to unexpected improvements in the wait-list control group. Controls also reduced weight as well as craving and improved on other outcomes. Second, the range of possible stimuli is narrower in smokers and drinkers, who often have a typical "drug of choice" (e.g., bottle of red wine; certain brand of cigarette). For eating, stimulus control is poor because even the same type of food (e.g., cake, cookies) can have many different shapes or colors. This assumption can be tested, however. For example, we would expect a greater reduction of weight and craving in overweight persons who imagine a wide range of unhealthy foods they like to eat during the retraining compared to those who imagined only a few unhealthy foods they like to eat (e.g., chips, pizza, and soda). Lastly, the imaginal retraining manual for drinkers gave additional recommendations that might have augmented the effect. However, no such tips were provided to smokers, so this cannot explain the efficacy gap in the study of smokers vs. the current study of people with overweight or obesity.

Motivation to engage the manual was higher than in smokers, but still $31.7 \%$ of the participants did not perform the exercises. Even though the technique is simple, other ways of presentation should be tested, such as video clips via social media demonstrating how to perform the exercises. The technique contains many elements. Future research should elucidate the differential effectiveness of its various elements and whether certain elements are dispensable, such as negative mood induction and the positive counter-sequence. Also, it would be helpful to know whether adding further behavioral tips to the manual or encouraging a special diet would enhance the effect.

The study is subject to a number of limitations. First, we do not know whether the effects are stable over time, and we have not yet tested long-term adherence to the technique. Relatedly, at this point we can only speculate which aspects of the eating behavior had changed (e.g., overall reduction or eating less unhealthy vs. eating more healthy food). Second, we relied on self-reports, which are prone to the effects of social desirability; future trials should monitor participants under more controlled conditions with objective assessments, including weighing with a scale $^{2}$. As in our forerunner trials, we refrained from implementing a sham condition for ethical reasons. Third, the efficacy of the technique needs to be explored in males as well, who have a higher prevalence of obesity, which seems to be more strongly related to alcohol consumption than in females ${ }^{48}$. Fourth, for exploratory purposes we did not control for multiple comparisons, which would have rendered many secondary outcomes non-significant. Fifth, future studies should explore motifs for non-adherence more rigorously (e.g., forgetting, inefficacy despite attempt to use it, no confidence in the rationale). Sixth, subsequent trials should test the present approach against an active control which may attenuate placebo and expectancy effects. Finally, whether the technique reduced craving and approach behavior as assessed by implicit measures has yet to be studied.

\section{Conclusions}

In individuals with overweight $(\mathrm{BMI}>25)$, many of whom met criteria for obesity, imaginal retraining significantly reduced craving for high-calorie food over a period of 6 weeks. A small to medium correlation emerged between utilization frequency and outcome. Treatment effects were larger in the subgroup encouraged to use electronic reminders than in the pooled retraining group. The long-term effects of the technique in terms of acceptance, feasibility, and effectiveness need to be tested as well as alternative ways of presenting the technique (e.g., video clips) and the long-term efficacy.

\section{Acknowledgements}

This study, as well as the work of S.K., was partially funded by the European Union (ERC-2016-StG-Self-Control-677804).

[2] We would like to thank one reviewer who suggested that those who had lost weight were perhaps more likely to recognize this and as a result both measured and reported their weight. 


\section{Author details}

'Department of Psychiatry and Psychotherapy, University Medical Center Hamburg-Eppendorf, Hamburg, Germany. ${ }^{2}$ Department of Occupational and Consumer Psychology, University of Freiburg, Freiburg, Germany. ${ }^{3} \mathrm{MSH}$ Medical School Hamburg, University of Applied Sciences and Medical University, Hamburg, Germany

\section{Conflict of interest}

The authors declare that they have no conflict of interest.

\section{Publisher's note}

Springer Nature remains neutral with regard to jurisdictional claims in published maps and institutional affiliations.

Received: 4 September 2019 Revised: 4 November 2019 Accepted: 4 November 2019

Published online: 28 November 2019

\section{References}

1. $\mathrm{Ng}, \mathrm{M}$. et al. Global, regional, and national prevalence of overweight and obesity in children and adults during 1980-2013: a systematic analysis for the Global Burden of Disease Study 2013. Lancet 384, 766-781 (2014).

2. Upadhyay, J., Farr, O., Perakakis, N., Ghaly, W. \& Mantzoros, C. Obesity as a disease. Med. Clin. North Am. 102, 13-33 (2018).

3. Marques, A., Peralta, M., Naia, A., Loureiro, N. \& de Matos, M. G. Prevalence of adult overweight and obesity in 20 European countries, 2014. Eur. J. Public Health 28, 295-300 (2018).

4. GBD 2015 Obesity Collaborators. et al. Health effects of overweight and obesity in 195 countries over 25 years. N. Engl. J. Med. 377, 13-27 (2017).

5. Puhl, R. \& Suh, Y. Stigma and eating and weight disorders. Curr. Psychiatry Rep. 17, 10 (2015).

6. Buchwald, $\mathrm{H}$. et al. Bariatric surgery: a systematic review and meta-analysis. JAMA 292, 1724 (2004).

7. Gloy, V. L. et al. Bariatric surgery versus non-surgical treatment for obesity: a systematic review and meta-analysis of randomised controlled trials. BMJ 347, $1-16$ (2013).

8. Bessesen, D. H. \& Van Gaal, L. F. Progress and challenges in anti-obesity pharmacotherapy. Lancet Diabetes Endocrinol. 6, 237-248 (2018).

9. Bechara, A. Decision making, impulse control and loss of willpower to resist drugs: a neurocognitive perspective. Nat. Neurosci. 8, 1458-1463 (2005).

10. Strack, F., Werth, L. \& Deutsch, R. Reflective and impulsive determinants of consumer behavior. J. Consum. Psychol. 16, 205-216 (2006).

11. JSBT, Evans \& Stanovich, K. E. Dual-process theories of higher cognition. Perspect. Psychol. Sci. 8, 223-241 (2013).

12. Kakoschke, N., Albertella, L., Lee, R. S. C. \& Wiers, R. W. Assessment of automatically activated approach-avoidance biases across appetitive substances. Curr. Addict. Rep. https://doi.org/10.1007/s40429-019-00254-2 (2019).

13. Kemps, E. \& Tiggemann, M. Approach bias for food cues in obese individuals. Psychol. Heal. 30, 370-380 (2015).

14. Varela, F. J., Thompson, E. \& Rosch, E. The Embodied Mind: Cognitive Science and Human Experience. (MIT Press, Cambridge, MA, 1991).

15. Wiers, R. W., Rinck, M., Kordts, R., Houben, K. \& Strack, F. Retraining automatic action-tendencies to approach alcohol in hazardous drinkers. Addiction 105, 279-287 (2010).

16. Kakoschke, N., Kemps, E. \& Tiggemann, M. Approach bias modification training and consumption: a review of the literature. Addict. Behav. 64, 21-28 (2017).

17. Eberl, C. et al. Approach bias modification in alcohol dependence: do clinical effects replicate and for whom does it work best? Dev. Cogn. Neurosci. 4 38-51 (2013).

18. Wiers, R. W., Eberl, C., Rinck, M., Becker, E. S. \& Lindenmeyer, J. Retraining automatic action tendencies changes alcoholic patients' approach bias for alcohol and improves treatment outcome. Psychol. Sci. 22, 490-497 (2011).

19. Fodor, L. A., Cosmoiu, A. \& Podina, I. R. Cognitive bias modification interventions for attention to and approach of appetitive food stimuli: a meta-analysis. J. Evid.-Based Psychother. 17, 85-104 (2017).
20. Ferentzi, $H$. et al. Retraining of automatic action tendencies in individuals with obesity: a randomized controlled trial. Appetite 126, 66-72 (2018).

21. Cristea, I. A., Kok, R. N. \& Cuijpers, P. The effectiveness of cognitive bias modification interventions for substance addictions: a meta-analysis. PLoS One 11, e0162226 (2016).

22. Boffo, M. et al. Cognitive bias modification for behavior change in alcohol and smoking addiction: Bayesian meta-analysis of individual participant data. Neuropsychol. Rev. 29, 52-78 (2019).

23. Boendermaker, W. J., Boffo, M. \& Wiers, R. W. Exploring elements of fun to motivate youth to do cognitive bias modification. Games Health J. 4, 434-443 (2015).

24. Moritz, S. et al. Imaginal retraining reduces alcohol craving in problem drinkers: a randomized controlled trial. J. Behav. Ther. Exp. Psychiatry https://doi.org/ 10.1016/j.jbtep.2019.04.001 (2019).

25. Fridland, E. \& Wiers, C. E. Addiction and embodiment. Phenomenol. Cogn. Sci. 17, 15-42 (2018).

26. Moritz, S. et al. Imaginal retraining reduces cigarette smoking: a randomized controlled study. 2019 (submitted).

27. Pudel, V. \& Westhöfer, J. Fragebogen zum Eßverhalten (FEV). (Hogrefe, Göttingen, 1989).

28. Meule, A., Hermann, T. \& Kübler, A. A short version of the food cravings questionnaire-trait: the FCQ-T-reduced. Front. Psychol. 5, 190 (2014).

29. Skevington, S. M., Lotfy, M. \& O'Connell, K. A. The World Health Organization's WHOQOL-BREF quality of life assessment: psychometric properties and results of the international field trial. A report from the WHOQOL Group. Qual. Life Res. 13, 299-310 (2004).

30. Göritz, A. S. Building and managing an online panel with phpPanelAdmin. Behav. Res. Methods 41, 1177-1182 (2009).

31. Göritz, A. S. Using online panels in psychological research. In: The Oxford Handbook of Internet Psychology (eds Joinson, A. N., McKenna, K. Y. A., Postmes, T. \& Reips, U. -D.) 473-485 (Oxford University Press, Oxford, UK, 2007).

32. Moritz, S. et al. We cannot change the past, but we can change its meaning. A randomized controlled trial on the effects of self-help imagery rescripting on depression. Behav. Res. Ther. 104, 74-83 (2018).

33. Cepeda-Benito, A. et al. The development and validation of Spanish versions of the State and Trait Food Cravings Questionnaires. Behav. Res. Ther. 38, 1125-1138 (2000).

34. Batra, P. et al. Relationship of cravings with weight loss and hunger. Results from a 6month worksite weight loss intervention. Appetite 69, 1-7 (2013).

35. Giel, K. E. et al. Effects of laparoscopic sleeve gastrectomy on attentional processing of food-related information: evidence from eye-tracking. Surg. Obes. Relat. Dis. 10, 277-282 (2014).

36. Hormes, J. M. \& Meule, A. Psychometric properties of the English Food Cravings Questionnaire-Trait-reduced (FCQ-T-r). Eat. Behav. 20, 34-38 (2016).

37. Meule, A. et al. On the differentiation between trait and state food craving: Half-year retest-reliability of the Food Cravings Questionnaire-Trait-reduced (FCQ-T-r) and the Food Cravings Questionnaire-State(FCQ-S). J. Eat. Disord. 2, 25 (2014).

38. Stunkard, A. J. \& Messick, S. The three-factor eating questionnaire to measure dietary restraint, disinhibition and hunger. J. Psychosom. Res. 29, 71-83 (1985).

39. Cappelleri, J. C. et al. Psychometric analysis of the Three-Factor Eating Questionnaire-R21: results from a large diverse sample of obese and non-obese participants. Int. J. Obes. 33, 611-620 (2009).

40. Beck, A. T., Steer, R. A. \& Brown, G. K. Beck Depression Inventory (2nd ed.). (The Psychological Corporation, San Antonio, TX, 1996).

41. Kühner, C., Bürger, C., Keller, F. \& Hautzinger, M. Reliabilität und Validität des revidierten Beck-Depressionsinventars (BDI-II). Befunde aus deutschsprachigen Stichproben [Reliability and validity of the revised Beck Depression Inventory (BDI-II). Findings from German-speaking samples]. Nervenarzt 78, 651-656 (2007).

42. Schmidt, J., Lamprecht, F. \& Wittmann, W. W. Zufriedenheit mit der stationären Versorgung. Entwicklung eines Fragebogens und erste Validitätsuntersuchungen [Content with inpatient care. Development of a questionnaire and a first study on validity]. Psychother. Psychosom. Med. Psychol. 39, 248-255 (1989).

43. Bray, G. A., Kim, K. K. \& Wilding, J. P. H. Obesity: a chronic relapsing progressive disease process. A position statement of the World Obesity Federation. Obes. Rev. 18, 715-723 (2017). 
44. Sumithran, P. et al. Long-term persistence of hormonal adaptations to weight loss. Obstet. Gynecol. Sur. 67, 91-92 (2012).

45. Dulloo, A. G. \& Montani, J.-P. Pathways from dieting to weight regain, to obesity and to the metabolic syndrome: an overview. Obes. Rev. 16, 1-6 (2015).

46. Mehl, N., Mueller-Wieland, L., Mathar, D. \& Horstmann, A. Retraining automatic action tendencies in obesity. Physiol. Behav. 192, 50-58 (2018).
47. Lüdtke, T., Pult, L. K., Schröder, J., Moritz, S. \& Bücker, L. A randomized controlled trial on a smartphone self-help application (Be Good to Yourself) to reduce depressive symptoms. Psychiatry Res. 269, 753-762 (2018).

48. Yeomans, M. R. Alcohol, appetite and energy balance: is alcohol intake a risk factor for obesity? Physiol. Behav. 100, 82-89 (2010). 